Ann. Génét. Sél. anim., I969, 1 (1), 67-70.

\title{
CALCUL DES INDEX DE SÉLECTION DANS LE GAS D'INDIVIDUS CONSANGUINS ET APPARENTÉS QUELCONQUES
}

\author{
R. ROUVIER \\ Station de Génétique quantitative et appliquée, \\ Centre national de Recherches zootechniques, 78 -Jouy-en-Joass. \\ Institut national de la Recherche agronomique
}

SOMMAIRE

Nous avons établi la formule de l'index de sélection pour un caractère à partir des valeurs phénotypiques de ce caractère observées chez cet individu et ses apparentés mesurés. Nous nous sommes placés dans le cas où les individus de l'échantillon considéré peuvent être consanguins et apparentés de façon quelconque, et pour un caractère dont la variation génétique est additive et de dominance.

La plupart des études concernant la théorie et le calcul des index de sélection massale, familiale ou combinée, se placent dans 1'hypothèse où les individus dont les phénotypes servent à établir l'index sont issus de parents non apparentés entre eux, et non consanguins. Cette hypothèse semble être peu réaliste dans certains cas de sélection animale et notamment dans celui des souches avicoles qui sont des populations fermées d'effectif limité; par ailleurs, dans celui des grands mammifères domestiques, l'utilisation de l'insémination artificielle conduit à une réduction de l'effectif génétique, et, en monte naturelle, on assiste fréquemment à l'utilisation d'un petit nombre de reproducteurs mâles par troupeau.

Quelques auteurs (LUSH, I947; JARDINE, I958; HENDERSON, 1963) ont abordé le problème de l'établissement des index de sélection pour des individus de populations consanguines, dans le cas particulier où la variance génétique est strictement additive. GILLOIS (I964) et HARRIs (I964) ont obtenu l'expression de la covariance génotypique entre deux individus pouvant être consanguins 
et apparentés de façon quelconque, lorsque l'on considère aussi la variance génétique de dominance. Leurs résultats permettent de proposer une méthode plus générale d'établissement des index de sélection, dans le cas d'une population consanguine. Ces deux auteurs ont considéré les quatre gènes situés aux quatre loci homologues de deux individus consanguins et apparentés quelconques $i$ et $j$. GrLıors a considéré les situations d'identité et de non identité de ces quatre gènes considérés simultanément, deux gènes étant identiques s'ils dérivent, par descendance mendélienne et sans mutation, d'un même gène ancêtre. Il a ainsi défini I5 situations d'identité, caractérisées par $I_{5}$ probabilités $\left(\delta_{1}\right.$ à $\left.\delta_{15}\right)$ appelées coefficients d'identité restreinte, et nous utiliserons par la suite ses notations pour l'expression de la covariance des valeurs génotypiques de deux individus apparentés. Cette covariance s'exprime à partir des probabilités indiquées ci-dessus et des composantes de la variance génétique de la population.

Nous prendrons ici comme exemple le cas de la sélection sur un seul caractère. Soit $\mathrm{P}_{\boldsymbol{i}}(i=\mathrm{I}$ à $n)$ la variable aléatoire phénotypique attachée à chaque individu numéro $i$ considéré. Nous écrivons :

$$
\mathrm{P}_{i}=Z_{i}+\mathrm{E}_{i}
$$

où $Z_{i}$ et $\mathrm{E}_{i}$ sont respectivement les variables aléatoires génotypiques et du milieu que nous supposons non corrélées, ce qui permet d'écrire :

$$
\operatorname{var} \mathrm{P}_{i}=\operatorname{var} Z_{i}+\operatorname{var} \mathrm{E}_{i}
$$

Nous nous plaçons dans les hypothèses où le caractère envisagé ne dépend que de gènes autosomaux et où, suivant le modèle classique, la variable aléatoire génotypique $Z_{t}$ est égale à la somme des deux variables aléatoires zygotiques $\mathrm{Y}_{i}$ et de dominance $\mathrm{D}_{i}$. De plus, nous supposons qu'il n'y a pas d'interactions entre loci non homologues (pas d'épistasie), et que les couples de loci homologues ajoutent indépendamment leurs contributions numériques à la valeur du caractère.

La sélection s'effectue parmi les individus d'un échantillon issu d'une population qui n'est pas en général connue dans sa totalité et elle doit conduire à maximiser l'espérance du progrès génétique $d \mathfrak{u}$ caractère considéré. Pour cela, nous utilisons le modèle que nous avons développé dans le cas général de la sélection sur plusieurs caractères (RovviER, 1969) mais qui concernait des individus non, consanguins et non apparentés de façon quelconque. Nous devons obtenir à partir des phénotypes observables de tous les individus de l'échantillon, des estimateurs non biaisés des valeurs génétiques additives $Y_{l}$ de chaque individu numéro $l$ et sélectionner la fraction des valeurs les plus élevées. Si les $P_{i}, Z_{i}, E_{i}$ suivent une loi normale à plusieurs variables, l'estimateur $I_{l}$ de $Y_{l}$ est l'équation de régression multiple de $\mathrm{Y}_{l}$ sur les $\mathrm{P}_{\mathfrak{l}}(i=\mathrm{I}, \ldots, l, \ldots n)$.

$$
\mathrm{I}_{l}=\sum_{i=1}^{i=n} \beta_{i}\left[\mathrm{P}_{i}-\mathrm{E}\left(\mathrm{P}_{i}\right)\right]
$$


Les $\beta_{i}$ peuvent être obtenus en résolvant le système d'équations suivant qui fait apparaître les variances et covariances théoriques de la population :

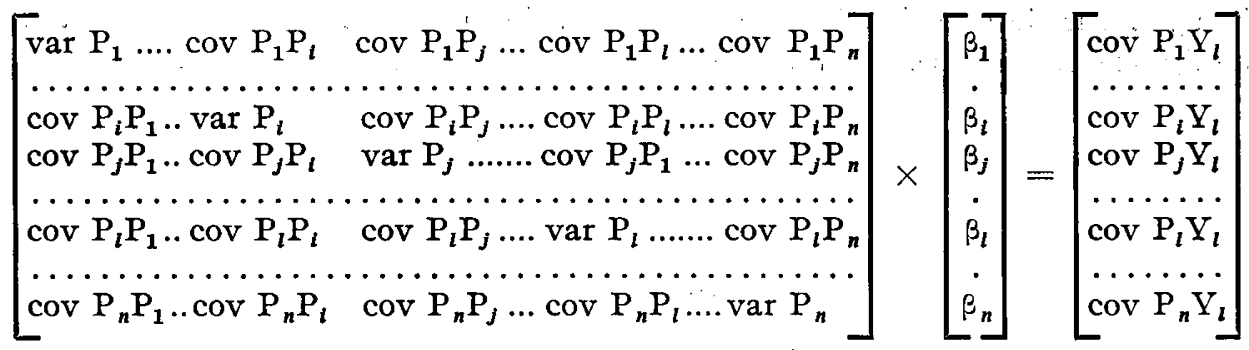

Nous avons, pour deux individus numéros $i$ et $j$ quelconques :

$$
\operatorname{cov} \mathrm{P}_{i} \mathrm{P}_{j}=\operatorname{cov} Z_{i} Z_{j}+\operatorname{cov} \mathrm{E}_{i} \mathrm{E}_{j}
$$

Les expressions des variances var $Z_{i}$ et des covariances cov $Z_{i} Z_{J}$ ont été obtenues par GILIoIS et HaRRIS pour des individus consanguins et apparentés quelconques. Il reste à calculer les termes de la forme $\operatorname{cov} \mathrm{P}_{l} \mathrm{Y}_{l}$ ainsi que cov $\mathrm{P}_{l} \mathrm{Y}_{l}$.

$$
\begin{aligned}
\operatorname{cov} \mathrm{P}_{i} \mathrm{Y}_{l} & =\operatorname{cov} Z_{i} \mathrm{Y}_{l}+\operatorname{cov} \mathrm{E}_{l} \mathrm{Y}_{l} \\
& =\operatorname{cov} Z_{l} \mathrm{Y}_{l}+\mathrm{O} \\
& =2 \varphi_{i l} 2 \mathrm{E}\left(\mathrm{X}_{1}^{2}\right)+\left(2 \delta_{1}+\delta_{2}+\delta_{3}\right) \mathrm{Ec}(\mathrm{XD})
\end{aligned}
$$

Le terme cov $\mathrm{E}_{l} \mathrm{Y}_{l}$ est nul par hypothèse. $\varphi_{i l}$ est égal au coefficient de parenté de MALECOT (I948). $\delta_{1}, \delta_{2}$ et $\delta_{3}$ sont des coefficients d'identité restreinte. $\delta_{1}$ est la probabilité d'identité des quatre gènes; $\delta_{2}$ et $\delta_{3}$ sont les probabilités d'identité de trois gènes (dont 2 pris chez l'individu $i$ et un chez l'individu $l$ ). $2 \mathrm{E}\left(\mathrm{X}^{2}\right)$ est la variance génétique additive et $E_{c}(X D)$ est l'espérance mathématique, conditionnée par l'identité des gènes, du produit des deux variables aléatoires gamétique et de dominance.

$$
\begin{aligned}
\operatorname{cov} \mathrm{P}_{l} \mathrm{Y}_{l} & =\operatorname{cov} Z_{l} \mathrm{Y}_{l}+\operatorname{cov} \mathrm{E}_{l} \mathrm{Y}_{l} \\
& =\operatorname{cov} Z_{l} \mathrm{Y}_{l}+\mathrm{O} \\
& =\left(\mathrm{I}+f_{l}\right) 2 \mathrm{E}\left(\mathrm{X}^{2}\right)+2 f_{l} \mathrm{Ec}(\mathrm{XD})
\end{aligned}
$$

où $f_{l}$ est le coefficient de consanguinité de l'individu numéro $l$.

Ces résultats indiquent que les formules classiques de la sélection des caractères polygéniques sont évidemment modifiées dans le cas d'apparentés quelconques consanguins. L'incidence numérique sur la valeur des coefficients de régression théoriques de la valeur génétique additive sur les phénotypes devrait cependant être étudiée.

L'utilisation pratique de ces formules nécessiterait par ailleurs d'obtenir des estimateurs suffisamment précis des diverses composantes de la variance génétique utilisées. Il faut noter enfin que, d'après HARRIS, l'utilisation de ces 
formules serait limitée à la première génération de sélection. Cet auteur indique en effet que les formules de la covariance entre apparentés consanguins quelconques, et de la variance génotypique, sont obtenues sous 1'hypothèse suivant laquelle il n'y a pas de sélection lors de l'obtention de la population actuelle. L'étude de l'influence de la sélection sur ces formules mériterait donc d'être approfondie.

Reçu pour publication en mars 1969.

\section{SUMMARY \\ CALCULATION OF SELECTION INDICES FOR INBRED INDIVIDUALS \\ AND ANY DEGREE OF RELATIONSHIP}

The formula for the selection index for a trait using the phenotypic value of the trait recorded on the individual and his measured relatives are developed. The case where the individuals in the sample studied can be inbred and related to any degree, and where the character has genetic variation only of the additive and dominance kinds are considered. The coefficients of the equation of the index are obtained, using a linear model, by calculating the regression of the individual's additive genetic value on the phenotypic values of himself and his relatives. The formulae for the index obtained up to now have been developed assuming that the genetic variance is all additive; the present study indicates how the dominance variance can be taken into account.

\section{RËFÉRENCES BIBLIOGRAPHIQUES}

Gricors M., 1964. Ia relation d'identité en génétique. Thèse Fac. Sci., Paris.

HARRIS D. L., I964. Genotypic covariances between inbred relatives. Genetics, 50, I3r9-1348.

HeNDERSON C. R., I963. Selection index and expected genetic advance. Statistical Genetics and Plant Breeding. Edited by W. D. Hanson and H. F. Robinson. Natl. Acad. Sci. Nat. Res. Council Publ., 982, $\mathrm{I}_{4} \mathrm{I}-\mathrm{I} 6_{3}$.

JARDINE R., 1958. Animal breeding and the estimation of genetic value. Heredity, 12, 499-5II.

L,USH J. H., 1947. Family merit and individual merit as bases for selection. Amer. Natur., 81, 24I-26r, $362-379$.

Malecor G., I948. Les mathématiques de l'hérédité. Masson et Cle, Paris.

Rouvier R., 1969. Contribution à l'étude des index de sélection sur plusieurs caractères. Thèse $3^{\mathbf{e}} c y c l e$, Fac. Sci., Paris. 